\title{
ATAC-ing the mechanisms of renin regulation
}

\author{
Steven D. Crowley \\ Renin-expressing cells have been conserved through evolution and \\ maintain blood pressure and fluid homeostasis. Lack of availability of \\ tools to study the specifics of renin regulation has limited advances in this \\ field. In the current issue of the Journal of Clinical Investigation, Martinez \\ and colleagues used the genome-wide assessment of the chromatin status \\ of cells and uncovered a unique set of super-enhancers that determine \\ the identity of renin cells. The renin super-enhancers play a key role in \\ the molecular memory of renin cell function, a mechanism at the core of \\ preserving homeostasis.
}

Department of Medicine, Duke University, Durham, North Carolina, USA. Department of Medicine, Durham VA Medical Centers, Durham, North Carolina, USA.

\section{Renin, the master regulator of blood pressure and fluid homeostasis}

As the rate-limiting protease that catalyzes the initial step in the activation of the renin angiotensin system (RAS) proteolytic cascade, renin is a master regulator of blood pressure and fluid homeostasis. Renin cleaves angiotensinogen to angiotensin I (Ang I), which is in turn converted by angiotensinconverting enzyme (ACE) to Ang II, the primary effector molecule of the RAS. Ang II then stimulates type 1 angiotensin receptors to execute several RAS functions that prevent circulatory collapse, including systemic vasoconstriction and salt and water reabsorption in the kidney nephron (1). Over the past 30 years, other constituents of the RAS cascade have been identified that temper the prohypertensive effects of $\mathrm{AT}_{1}$ receptors, including the enzyme ACE2, the effector molecule Ang 1-7, and the type 2 Ang II receptor (2-4). Moreover, the RAS is now known to play additional vital roles in biological functions as diverse as development, oncogenesis, inflammation, and even dementia (5-10). Nevertheless, despite the increasing complexity of the RAS, renin remains the ultimate driver of RAS activation with all of its pleiotropic functions.

Renin is highly conserved across multiple species from humans to Zebrafish (1). In humans, the renin gene rests on chromosome 1 and encodes a 406 amino acid renin precursor that is cleaved at the $\mathrm{C}$-terminus to yield prorenin and then at the N-terminus to form active renin (11). In recent years, a distinct receptor for prorenin (PRR) has been identified that highlights the capacity of the RAS to regulate development, blood pressure, and water homeostasis independently of its traditional effector molecule Ang II (12-15). While renin is expressed in several tissues $(16,17)$, renin derived from the kidney is thought to direct most of the key functions of the intrarenal RAS. In the healthy adult, renin is expressed in specialized cells termed juxtaglomerular (JG) cells, as they rest within the wall of the afferent arteriole where it abuts the kidney glomerulus. Sensitive methods have also detected renin expression at lower levels in the collecting tubule (18). Following a hypotensive insult, renin expression upregulates dramatically to activate the RAS $(19,20)$, thereby restoring blood pressure via preservation of intravascular volume and enhancement of vascular reactivity. This induction of renin relies in part on the "memory" of cells in the kidney that produced renin during development and can reinitiate renin transcription outside of the juxtaglomerular apparatus in response to a stressor. Nevertheless, although renin regulates a

Delated Article: p. 4787

Conflict of interest: The author has declared that no conflict of interest exists.

Reference information: / Clin Invest. 2018;128(11):4748-4750. https://doi.org/10.1172/JCI124177.

multitude of functions in the healthy and diseased organism, surprisingly little has been known to date regarding how cells with renin memory are invited to again produce renin when an organism experiencing hemorrhage or some other form of hypovolemia requires vigorous RAS activation for its survival. Indeed, research in this area was limited in the past because the tools to incisively explore the regulation of renin induction were not available.

\section{Molecular triggers of renin induction}

In the current issue of the Journal of Clinical Investigation, Martinez and colleagues have shed considerable light on the molecular triggers of renin upregulation by applying a sophisticated and complementary set of genetic interrogation techniques to three separate sets of murine cells with an active renin promoter $\left(\operatorname{Ren} 1^{c}\right)$ : (a) true JG cells isolated from animals expressing YFP under the control of Ren1', (b) renin-producing tumor cells (As4.1), and (c) cells with $\operatorname{Ren}^{c}$ activation due to renin deficiency, isolated by flow from the afferent arterioles of $\operatorname{Ren}^{1-/-}$ Ren $1^{\text {c-YFP+ }}$ animals (termed recruited cells) (21). These studies examined regions of the chromatin that regulate the capacity of renin cell descendants to transform into renin-producing cells during pathologic stress. For example, the assay for transposaseaccessible chromatin with high-throughput sequencing (ATAC-Seq) revealed regions of heightened chromatin accessibility with regulatory sequences occupied by the H3K27ac acetylated form of histone $\mathrm{H} 3$ as a marker of enhancer activation. As a complementary approach, ChIP-Seq analysis for the transcription coactivator mediator subunit 1 (Med1) and RNA polymerase II (RNA Pol II) identified enhancer regions that were common or unique to the three cell types. Finally, use of ranking ordering of super-enhancers (ROSE) software to identify super-enhancers isolated 211 such sequences (Figure 1). These super-enhancers are characterized by large H3cK27ac domains; 107 were 


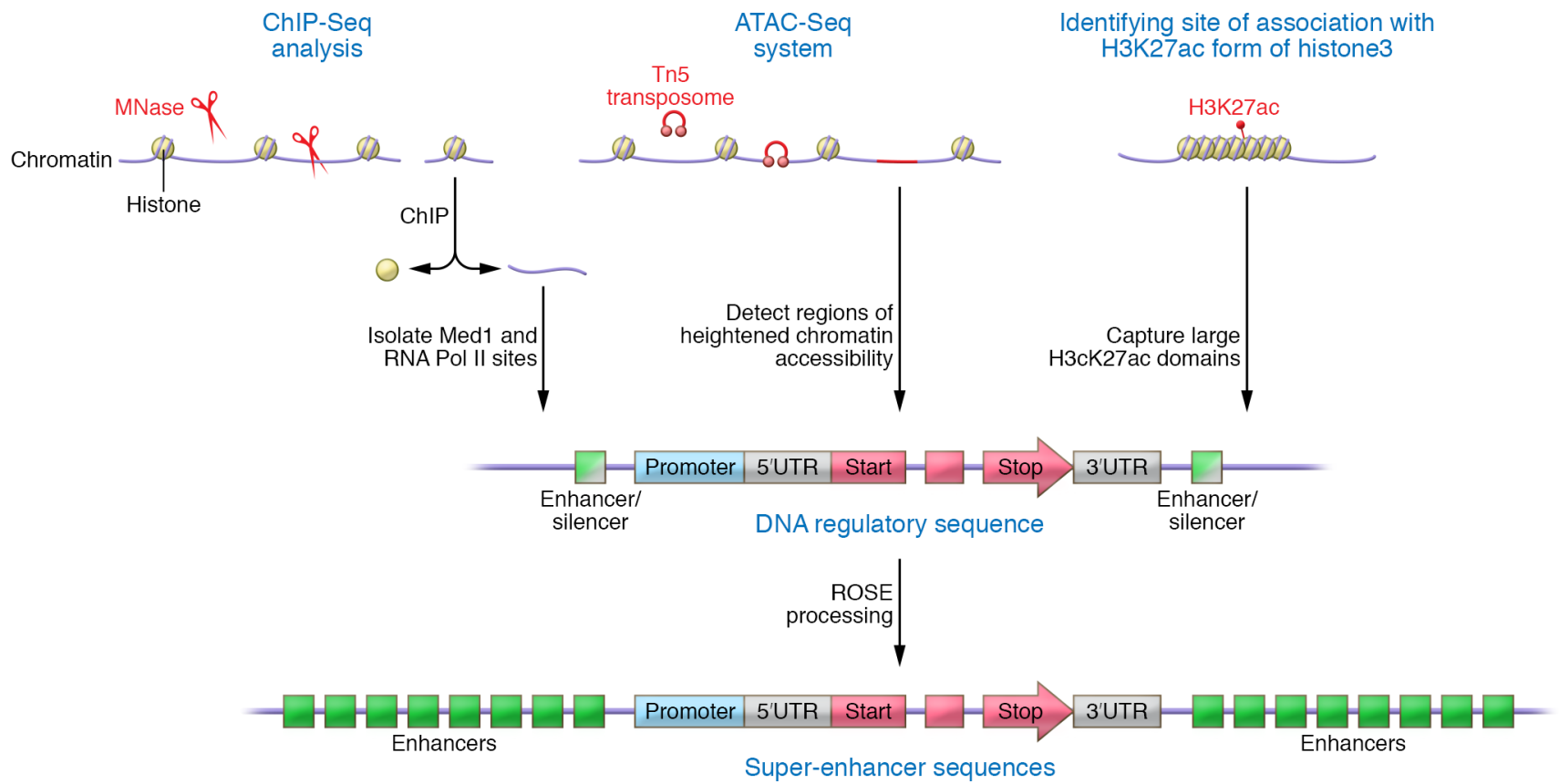

Figure 1. Strategy to identify super-enhancers via complementary methodologies. ATAC-Seq identifies regulatory sequences with heightened chromatin accessibility. Sites of association with H3K27ac histones mark regions of enhancer activation. ChIP-Seq analysis localizes sites for Med1 and RNA Pol II attachment. These characteristics are analyzed via ROSE software to identify super-enhancer regions that can be interrogated for their inclusion of novel regulatory genes that influence transcription of a putative target gene.

exclusive to the recruited cell population, whereas 91 were restricted to JG and As4.1 cells and may therefore identify active rather than recruited renin cells. Gene ontogeny analysis of these 91 genes confirmed the importance of several genes on the Notch and cAMP pathways to renin cell identity (21), some of which had been identified by the Gomez group in previous publications $(22,23)$.

Collectively, these studies confirm a super-enhancer region proximal to the renin gene, and analysis of predicted binding sites within this super-enhancer region identified several transcription factors known to regulate renin or vasculogenesis but also other novel regulatory genes. Accordingly, the Martinez et al. manuscript considerably advances our knowledge of the mechanisms through which the molecular memory for renin production can be tapped in settings of hemodynamic stress (21).

\section{The power of genomic tools}

More broadly, the Martinez et al. experiments illustrate the power of emerging genomic tools to precisely confirm or refute earlier biological discoveries, much like new DNA evidence at an old crime scene, but also to identify regulatory sequences impacting gene transcription that older, less-sensitive methods could not detect. Thus, while the researchers were aware that Notch and Rbpj are key regulators of renin transcription, these new studies pinpointed several new genes, such as Junb and the gene encoding thyroid hormone receptor alpha (THRA), that will require focused interrogation for their roles in renin induction. Given the recognized difficulties in reproducing and translating so many basic science findings for the benefit of patients (24), a paradigm in which new technologies verify the accuracy of previous reports while identifying novel lines of investigation is welcome and necessary.

Lastly, the studies from Martinez et al. fit nicely into a constellation of recent studies through which powerful genomic strategies are at long last revolutionizing the study of nephrology. With single-cell transcriptomics, we can now define key cell lineages and their precursors within the kidney involved in normal renal physiology and in disease $(25,26)$. When applied to a kidney biopsy, single-cell genomic analysis can yield information to facilitate the advent of precision medicine (27). When combined with super-enhancer analysis, single-cell transcriptomics may predict which cell lineages are primed to become involved in renal damage before severe disease emerges, thus providing a desperately needed ounce of prevention for the nephrology field. For example, since hypertension remains uncontrolled in up to $50 \%$ of patients and is associated with catastrophic complications, performing a kidney biopsy in an otherwise healthy patient with prehypertension would become considerably more relevant if it revealed whether the patient would develop high renin hypertension in the future and which genes should be targeted to prevent renin induction. Similarly, in diabetic nephropathy where RAS activation plays such a detrimental role in disease progression, the kidney biopsy could reemerge as a powerful tool if genomic tools could elucidate the mechanisms that are provoking RAS stimulation in individual patients. Accordingly, new genomic tools can transform the application of older, blunt diagnostic tests like the kidney biopsy. Accordingly, the application of complementary genomic strategies now offers the promise of long-awaited specific therapies for renal disease. Thus, one message from the studies from Martinez et al. viewed in the context of other recent investigations employing novel genomic interrogation strategies is that there has never been a more excit- 
ing time at which to enter and remain in nephrology research.

\section{Acknowledgments}

This work was supported by NIH grants DK087893 and HL128355, and by the Veterans Health Administration, Office of Research and Development, Biomedical Laboratory Research and Development Grant BX000893.

Address correspondence to: Steven D. Crowley, Division of Nephrology, Department of Medicine, Durham VA, and Duke University Medical Centers, DUMC Box 103015, Durham, North Carolina 27710, USA. Phone: 919.684.9788; Email: steven. d.crowley@duke.edu.

1. Sparks MA, Crowley SD, Gurley SB, Mirotsou M, Coffman TM. Classical Renin-Angiotensin system in kidney physiology. Compr Physiol. 2014;4(3):1201-1228.

2. Gurley SB, et al. Altered blood pressure responses and normal cardiac phenotype in ACE2-null mice. J Clin Invest. 2006;116(8):2218-2225.

3. Ferrario CM, et al. Angiotensin-(1-7): a new hormone of the angiotensin system. Hypertension. 1991;18(5 suppl):III126-III133.

4. Carey RM, Wang ZQ, Siragy HM. Role of the angiotensin type 2 receptor in the regulation of blood pressure and renal function. Hypertension. 2000;35(1 pt 2):155-163.

5. Oliverio MI, et al. Reduced growth, abnormal kidney structure, and type 2 (AT2) angiotensin receptor-mediated blood pressure regulation in mice lacking both AT1A and AT1B receptors for angiotensin II. Proc Natl Acad Sci U S A.
1998;95(26):15496-15501.

6. Shen XZ, et al. Mice with enhanced macrophage angiotensin-converting enzyme are resistant to melanoma. Am J Pathol. 2007;170(6):2122-2134.

7. Egami K, et al. Role of host angiotensin II type 1 receptor in tumor angiogenesis and growth. JClin Invest. 2003;112(1):67-75.

8. Muller DN, et al. NF- $\mathrm{kB}$ inhibition ameliorates angiotensin II-induced inflammatory damage in rats. Hypertension. 2000;35(1):193-201.

9. Guzik TJ, et al. Role of the T cell in the genesis of angiotensin II induced hypertension and vascular dysfunction. JExp Med. 2007;204(10):2449-2460.

10. Faraco G, et al. Perivascular macrophages mediate the neurovascular and cognitive dysfunction associated with hypertension. J Clin Invest. 2016;126(12):4674-4689.

11. Hobart PM, Fogliano M, O'Connor BA, Schaefer IM, Chirgwin JM. Human renin gene: structure and sequence analysis. Proc Natl Acad Sci US A. 1984;81(16):5026-5030.

12. Nguyen G, Delarue F, Burcklé C, Bouzhir L, Giller T, Sraer JD. Pivotal role of the renin/ prorenin receptor in angiotensin II production and cellular responses to renin. J Clin Invest. 2002;109(11):1417-1427.

13. Cruciat CM, et al. Requirement of prorenin receptor and vacuolar $\mathrm{H}^{+}$-ATPase-mediated acidification for Wnt signaling. Science. 2010;327(5964):459-463.

14. Ramkumar N, et al. Collecting duct principal, but not intercalated, cell prorenin receptor regulates renal sodium and water excretion. Am JPhysiol Renal Physiol. 2018;315(3):F607-F617.

15. Wang $\mathrm{F}$, et al. Antidiuretic action of collecting duct (Pro)renin receptor downstream of vasopressin and PGE2 receptor EP4. JAm Soc Nephrol. 2016;27(10):3022-3034.

16. Field LJ, McGowan RA, Dickinson DP, Gross KW. Tissue and gene specificity of mouse renin expression. Hypertension. 1984;6(4):597-603.
17. Sigmund CD, Jones CA, Fabian JR, Mullins JJ, Gross KW. Tissue and cell specific expression of a renin promoter-reporter gene construct in transgenic mice. Biochem Biophys Res Commun. 1990;170(1):344-350.

18. Prieto-Carrasquero MC, et al. Enhancement of collecting duct renin in angiotensin IIdependent hypertensive rats. Hypertension. 2004;44(2):223-229.

19. Gomez RA, et al. Recruitment of renin geneexpressing cells in adult rat kidneys. Am J Physiol. 1990;259(4 pt 2):F660-F665.

20. Crowley SD, et al. Angiotensin II causes hypertension and cardiac hypertrophy through its receptors in the kidney. Proc Natl Acad Sci US A. 2006;103(47):17985-17990.

21. Martinez MF, et al. Super-enhancers maintain renin-expressing cell identity and memory to preserve multi-system homeostasis. JClin invest. 2018;128(11):4787-4803.

22. Brunskill EW, et al. Genes that confer the identity of the renin cell. J Am Soc Neph. 2011;22(12):2213-2225.

23. Castellanos Rivera RM, et al. Transcriptional regulator RBP-J regulates the number and plasticity of renin cells. Physiol Genom. 2011;43(17):1021-1028.

24. Begley CG, Ioannidis JP. Reproducibility in science: improving the standard for basic and preclinical research. Circ Res. 2015;116(1):116-126.

25. Park J, et al. Single-cell transcriptomics of the mouse kidney reveals potential cellular targets of kidney disease. Science. 2018;360(6390):758-763.

26. Lindström NO, et al. Conserved and divergent features of mesenchymal progenitor cell types within the cortical nephrogenic niche of the human and mouse kidney. J Am Soc Nephrol. 2018;29(3):806-824.

27. $\mathrm{Wu} \mathrm{H}$, et al. Single-cell transcriptomics of a human kidney allograft biopsy specimen defines a diverse inflammatory response. J Am Soc Nephrol. 2018;29(8):2069-2080. 